COLORECTAL CANCER

\title{
The prognostic significance of K-ras, p53, and APC mutations in colorectal carcinoma
}

\author{
A Conlin, G Smith, F A Carey, C R Wolf, R J C Steele
}

Gut 2005;54:1283-1286. doi: 10.1136/gut.2005.066514

See end of article for authors' affiliations

.....................

Correspondence to: Dr A Conlin, Biomedical Research Centre, Level 5 Ninewells Hospital and Medical School, Ninewells Ave, Dundee DD1 9SY, UK; abbyconlin75@ yahoo.co.uk

Revised version received 11 April 2005

Accepted for publication 12 April 2005

Published online first

20 April 2005
Background: Accumulation of molecular alterations, including mutations in Kirsten-ras (K-ras), p53, and adenomatous polyposis coli (APC), contribute to colorectal carcinogenesis. Our group has previously characterised a panel of sporadic colorectal adenocarcinomas for mutations in these three genes and has shown that p53 and K-ras mutations rarely occur in the same colorectal tumour. This suggests that mutations in these genes are on separate pathways to colorectal cancer development and may influence patient prognosis independently.

Aims: To correlate the presence or absence of mutations in K-ras, p53, and APC with survival in a cohort of colorectal cancer patients.

Patients: A series of 107 inpatients treated surgically for colorectal cancer in Tayside, Scotland between November 1997 and December 1999.

Methods: Colorectal tumours were characterised for mutations in K-ras, p53, and APC. Kaplan-Meier survival curves were constructed using overall survival and disease specific survival as the primary end points. Patient survival was analysed using the log rank test and Cox proportional hazards model.

Results: Patients with K-ras mutations had significantly poorer overall survival than patients without K-ras mutations $(p=0.0098)$. Multivariate analysis correcting for Dukes' stage, age, and sex confirmed this (hazard ratio 2.9 (95\% confidence interval 1.4-6.2); $p=0.0040$ ). K-ras mutations were also significantly associated with poorer disease specific survival. The presence of APC and p53 mutations did not affect survival in this cohort of patients ( $p=0.9034$ and $p=0.8290$, respectively).

Conclusions: Our data indicate that the presence of K-ras mutations predicts poor patient prognosis in colorectal cancer, independently of tumour stage.
C olorectal cancer is a significant cause of morbidity and mortality in the developed world with over 30000 new cases diagnosed annually in the UK. ${ }^{1}$ Although patients diagnosed with early stage disease have a high cure rate, many present later when five year survival is poor. It has long been recognised that colorectal carcinogenesis is a multistep process involving accumulation of molecular alterations ${ }^{2}$ and it has also been suggested that associations may exist between many of these abnormalities and patient survival. ${ }^{34}$ Increased understanding of the changes in specific molecular pathways that are responsible for disease progression and poor prognosis may prove essential in the development of more effective targeted treatment.

It has been suggested that progression from benign colorectal adenoma to malignant carcinoma arises from an accumulation of several events, including chromosomal abnormalities, genetic mutations, and epigenetic changes. ${ }^{5-7}$ These changes result in inactivation of tumour suppressor genes and DNA mismatch repair genes or activation of oncogenes. Mutation of the genes adenomatous polyposis coli (APC), Kirsten-ras (K-ras), and p53 are thought to be critical in the development of colorectal cancer. ${ }^{8}$

APC is a tumour suppressor gene encoding a large $312 \mathrm{kDa}$ protein with an important role in the wnt signalling pathway, intercellular adhesion, cytoskeleton stabilisation, cell cycle regulation, and apoptosis. ${ }^{9-13}$ Inactivating, predominantly truncating, mutations of APC are thought to allow unregulated transcription of oncogenes such as c-myc and cyclin Dl, therefore promoting tumorigenesis. ${ }^{14} 15$

The p53 tumour suppressor gene encodes a nuclear phosphoprotein with the ability to bind directly to DNA and act as a transcriptional activator. Genes activated by p53 cause cell cycle arrest allowing a damaged cell to either repair itself or be targeted for apoptosis. ${ }^{16}$ Mutations of the p53 gene are among the commonest genetic alterations in all cancers.

K-ras is part of a group of three highly homologous oncogenes and encodes a small $21 \mathrm{kDa}$ protein (p2lras) involved in transduction of external stimuli to effector molecules across plasma membranes..$^{17}$ This protein has intrinsic GTPase activity, allowing inactivation following signal transduction in the normal cellular environment. Activating mutations of K-ras occurring early in colorectal tumorigenesis are thought to abolish GTPase activity, leading to increased and unregulated cellular proliferation and malignant transformation.

Our group has characterised a consecutive cohort of 107 colorectal adenocarcinomas for mutations in APC, K-ras, and p53 showing that simultaneous mutations in all three genes was uncommon in the same tumour and, in particular, mutations in p53 and K-ras rarely occurred together (fig l). ${ }^{18}$ This has important implications as it suggests that K-ras and p53 mutations are on separate pathways to colorectal tumorigenesis and are not part of a common pathway of accumulating genetic change. Although K-ras mutations in colorectal cancer have been associated with poor prognosis in several publications, including a large multicentre study, ${ }^{19}$ the literature on p53 mutations and prognosis is controversial, with some studies reporting a link with poor prognosis ${ }^{20}$ and some reporting no association. ${ }^{21}$

We have completed a follow up database of this patient cohort and analysed K-ras, p53, and APC mutations in association with survival. To the best of our knowledge, this is the first study to examine the relationship between patient

Abbreviations: K-ras, Kirsten-ras; APC, adenomatous polyposis coli; $M C R$, mutation cluster region; $H R$, hazard ratio 


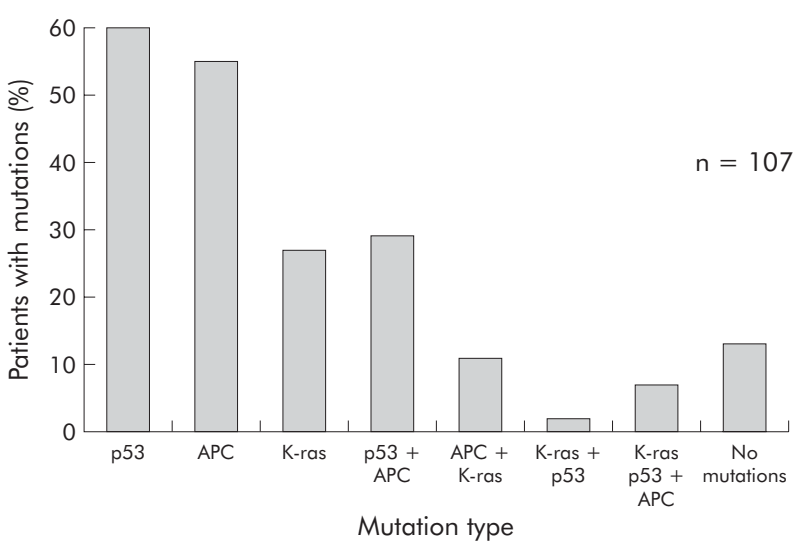

Figure 1 Percentage of patients $(n=107)$ with mutations in $p 53$ $(n=64)$, adenomatous polyposis coli (APC) $(n=59)$, Kirsten-ras (K-ras) $(n=29), p 53+\operatorname{APC}(n=31), A P C+K$-ras $(n=12), K$-ras + p53 $(n=2)$, $\mathrm{K}$-ras $+\mathrm{p} 53+\mathrm{APC}(\mathrm{n}=7)$, and no mutations in K-ras, APC, or p53 $(n=14)$. Only a small percentage of patients had a mutation in both $p 53$ and $\mathrm{K}$-ras and these mutations co-occurred less frequently than expected by chance ( $p<0.01$, Fisher's exact test). ${ }^{18}$ This suggests that mutations in these genes are on separate pathways to colorectal tumorigenesis.

prognosis and mutations in K-ras, p53, and APC in a single cohort of colorectal cancer patients.

\section{METHODS}

\section{Patients}

Patients undergoing surgery for colorectal cancer at Ninewells Hospital, Dundee, or Perth Royal Infirmary between November 1997 and December 1999 were enrolled in this study. All participants were Caucasian, aged 4580 years, and did not have a previous history of cancer. There were 42 female and 65 male patients. This study was approved by the Tayside Committee for Medical Research Ethics.

\section{Samples}

Resected colon specimens were transported directly from theatre to the pathology department and examined by a consultant pathologist (FAC) who selected normal and tumour tissue samples. A total of 107 samples were stored in liquid nitrogen for later analysis. DNA was extracted, amplified, and sequenced from fresh frozen tumour tissue, as described below.

\section{Mutation detection}

Genomic DNA was extracted from each tumour tissue sample and regions of each gene were amplified using specific oligonucleotide primers. Specifically, the mutation cluster region (MCR) of APC, codons 12, 13, and 61 of K-ras, and the entire coding region of p53 were examined. Over $60 \%$ of all somatic mutations in APC occur within the MCR (codons 1286-1513) and most K-ras mutations occur at codon 12, 13, or 61. Although most mutations in p53 occur within exons 5-8 at "hotspot" codons 175, 245, 248, and 273, the MCR is less well characterised so the entire coding region was analysed. Mutation detection was carried out using a combination of denaturing high performance liquid chromatography and direct DNA sequencing. These methods have been previously described in detail. ${ }^{18}$

\section{Statistics}

Kaplan-Meier survival curves were constructed for overall and disease specific survival and the log rank test was used to evaluate differences between survival curves of patients with and without genetic mutations. Survival was defined as the time from the date of diagnosis of colorectal cancer to the date of death. Multivariate analysis was carried out using the Cox proportional hazards model with hazard ratios (HRs) and 95\% confidence intervals (CI) being calculated for each model. Models correcting for disease stage (Dukes' stage), patient age at diagnosis, and sex were developed. Dukes' stage was treated as a categorical variable with each stage compared relative to Dukes' A. All analyses were carried out using the Statistical Package for the Social Services (SPSS) software package (Chicago, Illinois, USA).

\section{RESULTS}

Clinical information on 107 patients was entered into the follow up database (table 1). Minimum and maximum follow up periods were 55 and 81 months, respectively, with a mean colorectal cancer patient survival of 58 months.

Analysis of the 107 tumours revealed mutation frequencies in p53 of $61 \%$, APC of $56 \%$, and K-ras of $27 \%$, as previously reported. ${ }^{18}$ These results are consistent with literature reports showing that APC was mutated in $50-83 \%$ of sporadic colorectal cancers, ${ }^{22-24}$ p53 was mutated in $41-69 \%,{ }^{3625}$ and $\mathrm{K}$-ras was mutated in $20-38 \% .^{26-29}$ Only $6 \%$ of tumours contained mutations in all three genes and mutations in both p53 and K-ras rarely occurred (fig 1). Most p53 mutations occurred in exons 5-8 and most APC mutations were frame shifts introducing a premature stop codon; $79 \%(n=23)$ of K-ras mutations occurred in codon 12 and $21 \%(n=6)$ in

\section{Table 1 Patient clinical characteristics}

\begin{tabular}{llll}
\hline Characteristic & Female & Male & Total \\
\hline No of patients & 42 & 65 & 107 \\
Age (median (range)) & $69(46-80)$ & $67(50-78)$ & 67 (46-80) \\
Dukes' stage & 4 & 14 & 18 \\
A & 15 & 17 & 32 \\
B & 20 & 25 & 45 \\
C & 3 & 9 & 12 \\
D & 5 & 11 & 16 \\
Tumour localisation & 20 & 26 & 46 \\
$\quad$ Right side & 17 & 28 & 45 \\
Left side & 25 & 35 & 60 \\
$\quad$ Rectal & 17 & 30 & 47 \\
Patients alive at last follow up & & & 39 \\
Patients dead at last follow up & 15 & 24 & 5 \\
Cause of death & 1 & 4 & 3 \\
$\quad$ Colorectal cancer & 1 & 2 & \\
$\quad$ Other & & & \\
$\quad$ Unknown & & &
\end{tabular}



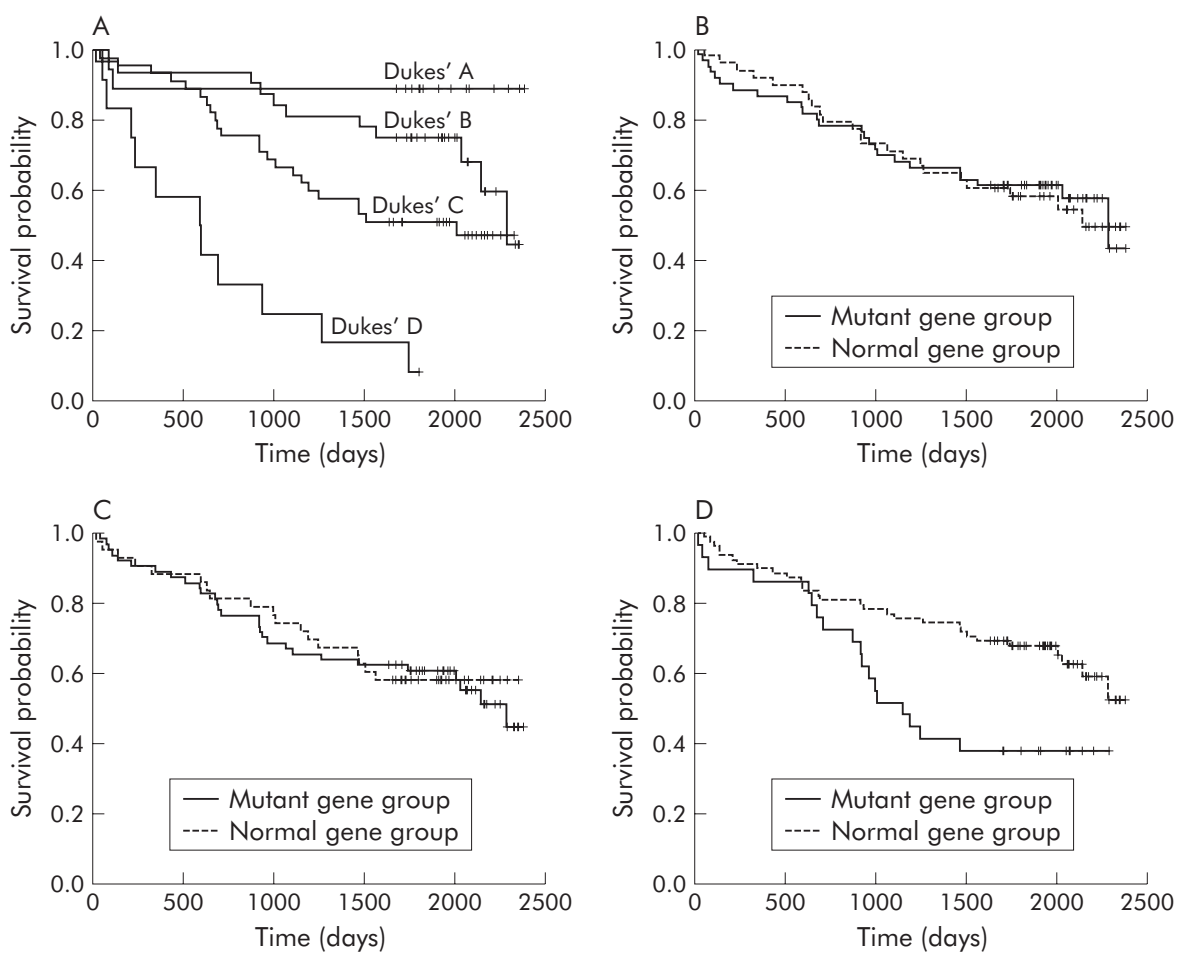

Figure 2 (A) Overall colorectal cancer survival stratified by Dukes' stage $(n=107)$. (B) Overall colorectal cancer survival analysed according to the presence $(n=59)$ or absence $(n=48)$ of an adenomatous polyposis coli (APC) mutation. APC mutation was not associated with survival in this panel of patients $(p=0.9034)$. (C) Overall colorectal cancer survival analysed according to the presence $(n=64)$ or absence $(n=43)$ of a p53 mutation. p53 mutation was not associated with survival $(p=0.8290)$. (D) Overall colorectal cancer survival analysed according to the presence $(n=29)$ or absence $(n=78)$ of a K-ras mutation. K-ras was significantly associated with poor prognosis in the cohort of patients $(p=0.0098)$. Hash marks on survival curves indicate censored cases.

codon 13 with most of these mutations being base pair transitions. No mutations in K-ras, codon 61 were found. ${ }^{18}$

Kaplan-Meier survival curves were constructed using overall survival from date of diagnosis as the primary end point (fig 2). Log rank statistics showed that K-ras mutations were significantly associated with poor patient prognosis $(\mathrm{p}=0.0098)$. Neither $\mathrm{p} 53$ or APC was found to affect prognosis $(p=0.8290$ and $p=0.9034$, respectively). Multivariant analysis using Cox regression and correcting for Dukes' stage, age at diagnosis, and sex showed that K-ras was an independent prognostic variable in this patient cohort (HR 2.9 (95\% CI 1.4-6.2); p = 0.0040). Dukes' stage (HR 2.6 $(95 \%$ CI $1.8-3.9) ; \mathrm{p}<0.0001)$ and T stage (HR 2.8 (95\% CI 1.8-4.3); $\mathrm{p}<0.0001$ ) were also shown to significantly affect prognosis. We also carried out analysis based on disease specific survival. Log rank statistic $(p=0.0380)$ and Cox regression correcting for Dukes' stage, age at diagnosis, and sex confirmed that K-ras mutations were associated with poor prognosis (HR 2.6 (95\% CI 1.1-6.0); $\mathrm{p}=0.0270$ ).

K-ras mutations occurred more commonly in patients with later stage disease, with 20 of the 29 K-ras mutations detected in patients with Dukes' stage C or D disease and 21 of the 29 mutations found in patients with T stage 3 or 4 .

\section{DISCUSSION}

Our group has characterised a large panel of colorectal cancers for mutations in APC, p53, and K-ras and shown that mutations in all three genes occurred uncommonly in the same colorectal tumour and that p53 and K-ras mutations rarely coexisted in the same tumour. ${ }^{18}$ This suggests that mutations in these genes are on separate pathways in colorectal tumorigenesis. We have now studied the clinical implications of these specific molecular changes in the original patient cohort and established that p53 and APC mutations do not affect survival. However, patients who have a K-ras mutation in their colorectal tumour have a significantly poorer prognosis than those without a K-ras mutation. A large proportion of patients with a K-ras mutation presented with late stage disease, and therefore to ensure that the prognostic effect associated with K-ras mutation was not just a reflection of this later stage disease, we carried out a multivariant analysis. We developed models correcting for disease stage, age, and sex and analysed the data using Cox regression. This confirmed that the presence of K-ras mutations was an independent adverse prognostic variable using overall and disease specific survival as primary end points. A multicentre study showed that only one of the 12 possible K-ras mutations appeared to infer poor prognosis in colorectal cancer. ${ }^{19}$ It was not possible to accurately assess the effect of specific K-ras mutations on survival in our patient cohort due to the small patients numbers in each group.

Mutated K-ras is constitutively active and we hypothesise that this may cause increased and unregulated signalling down pathways normally involved in growth and differentiation such as the mitogen activated kinase pathway. ${ }^{30}$ Our data suggest that K-ras mutation is a marker of aggressive tumour phenotype, and therefore detecting this mutation at an earlier disease stage may be of importance. This could also have significant implications for treatment, as patients with Dukes' stage A and B tumours undergo surgery with curative intent but are not routinely offered adjuvant therapy. ${ }^{31}$ Patients with early stage disease and a K-ras mutation in their tumour may benefit from an alternative more aggressive treatment regimen.

Randomised controlled trials and pilot programmes have demonstrated that screening with guaiac based faecal occult blood testing reduces disease specific mortality and is feasible 
within the UK National Health Service. ${ }^{32} 33$ A national colorectal cancer screening programme is to be implemented and will increase the proportion of the population with Dukes' stage A and B cancers. It is inevitable that some of the screen detected early stage cancers will have an aggressive phenotype and more sophisticated measurements of tumour behaviour will be required in order to utilise adjuvant treatment in an appropriate manner. Our data suggest that mutation detection may provide information that could allow tailoring of treatment in a situation where Dukes' staging may be misleading. K-ras appears to be a suitable candidate gene requiring further evaluation in this context.

An important aspect of this study is that for the first time we have analysed all three major gene mutations in colorectal cancer in relation to prognosis in a single patient cohort. The results of this study would indicate that, while APC and p53 mutations do not have clinical significance, mutation of K-ras defines a subgroup of cancers that arise independently of p53 mutation, tend to be of advanced stage at presentation, and are associated with an adverse prognosis at all disease stages.

\section{ACKNOWLEDGEMENTS}

This work was completed with funding from the Food Standards Agency (contracts TO1004 and TO1022) and in collaboration with "The Colorectal Cancer Study Group".[34]

\section{Authors' affiliations}

A Conlin, Biomedical Research Centre, Ninewells Hospital and Medical School, Dundee, UK

G Smith, Biomedical Research Centre, University of Dundee, Dundee, UK

F A Carey, Department of Pathology, University of Dundee, UK C R Wolf, CRUK Molecular Pharmacology Unit, Biomedical Research Centre, University of Dundee, Dundee, UK

R J C Steele, Departments of Surgery and Molecular Oncology, University of Dundee, Dundee, UK

Conflict of interest: None declared.

\section{REFERENCES}

1 Cancer incidence in five continents, vol VIII. IARC Sci Publ 2002;155:1781.

2 Vogelstein B, Fearon ER, Hamilton SR, et al. Genetic alterations during colorectal-tumor development. N Engl J Med 1988;319:525-32.

3 Hamelin R, Laurent-Puig $\mathrm{P}$, Olschwang $\mathrm{S}$, et al. Association of $\mathrm{p} 53$ mutations with short survival in colorectal cancer. Gastroenterology 1994;106:42-8.

4 Ahnen DJ, Feigl P, Quan G, et al. Ki-ras mutation and p53 overexpression predict the clinical behavior of colorectal cancer: a Southwest Oncology Group study. Cancer Res 1998;58:1149-58.

5 Leslie A, Pratt NR, Gillespie K, et al. Mutations of APC, K-ras, and p53 are associated with specific chromosomal aberrations in colorectal adenocarcinomas. Cancer Res 2003;63:4656-61.

6 Baker SJ, Preisinger AC, Jessup JM, et al. p53 gene mutations occur in combination with 17p allelic deletions as late events in colorectal tumorigenesis. Cancer Res 1990;50:7717-22.

7 Herman JG, Umar A, Polyak K, et al. Incidence and functional consequences of hMLH1 promoter hypermethylation in colorectal carcinoma. Proc Natl Acad Sci U S A 1998;95:6870-5.

8 Fearon ER, Vogelstein B. A genetic model for colorectal tumorigenesis. Cell 1990;61:759-67.
9 Miller JR, Hocking AM, Brown JD, et al. Mechanism and function of signal transduction by the $\mathrm{Wnt}_{\mathrm{nt}} /$ beta-catenin and $\mathrm{Wnt} / \mathrm{Ca} 2+$ pathways. Oncogene 1999; 18:7860-72.

10 Jou TS, Stewart DB, Stappert J, et al. Genetic and biochemical dissection of protein linkages in the cadherin-catenin complex. Proc Natl Acad Sci U S A 1995;92:5067-71.

11 Kawasaki Y, Senda T, Ishidate T, et al. Asef, a link between the tumor suppressor APC and G-protein signaling. Science 2000;289:1194-7.

12 Morrison EE, Wardleworth BN, Askham JM, et al. EB1, a protein which interacts with the APC tumour suppressor, is associated with the microtubule cytoskeleton throughout the cell cycle. Oncogene 1998;17:3471-7.

13 Morin PJ, Vogelstein B, Kinzler KW. Apoptosis and APC in colorectal tumorigenesis. Proc Natl Acad Sci U S A 1996;93:7950-4.

14 He TC, Sparks AB, Rago C, et al. Identification of c-MYC as a target of the APC pathway. Science 1998;281:1509-12.

15 Tetsu O, McCormick F. Beta-catenin regulates expression of cyclin D1 in colon carcinoma cells. Nature 1999;398:422-6.

16 Steele RJC, Thompson AM, Hall PA, et al. The p53 tumour suppressor gene. Br J Surg 1998;85:1460-7.

17 Bos JL. Ras oncogenes in human cancer: a review. Cancer Res 1989:49:4682-9.

18 Smith G, Carey FA, Beattie J, et al. Mutations in APC, Kirsten-ras, and p53alternative genetic pathways to colorectal cancer. Proc Natl Acad Sci U S A 2002;99:9433-8.

19 Andreyev HJN, Norman AR, Cunningham D, et al. Kirsten ras mutations in patients with colorectal cancer: the 'RASCAL II' study. Br J Cancer 2001;85:692-6.

20 Goh HS, Yao J, Smith DR. p53 point mutation and survival in colorectal cancer patients: effect of disease dissemination and tumour location. Int J Oncol 1999; 15:491-8.

21 Soong R, Powell B, Elsaleh $\mathrm{H}$, et al. Prognostic significance of TP53 gene mutation in 995 cases of colorectal carcinoma. Influence of tumour site, stage, adjuvant chemotherapy and type of mutation. Eur J Cancer 2000;36:2053-60

22 Powell SM, Zilz N, Beazer-Barclay Y, et al. APC mutations occur early during colorectal tumorigenesis. Nature 1992;359:235-7.

23 Rowan AJ, Lamlum $\mathrm{H}$, llyas $\mathrm{M}$, et al. APC mutations in sporadic colorectal tumors: A mutational "hotspot" and interdependence of the "two hits". Proc Natl Acad Sci U S A 2000;97:3352-7

24 De Filippo C, Luceri C, Caderni G, et al. Mutations of the APC gene in human sporadic colorectal cancers. Scand J Gastroenterol 2002;37:1048-53

25 Slebos RJC, Baas 10 , Clement $M$, et al. Clinical and pathological associations with $\mathrm{p} 53$ tumour-suppressor gene mutations and expression of $\mathrm{p} 21$ WAF1/Cip1 in colorectal carcinoma. Br J Cancer 1996;74:165-71.

26 Andreyev HJN, Norman AR, Cunningham D, et al. Kirsten ras mutations in patients with colorectal cancer: the multicenter "RASCAL" study. J Nat Cancer Inst 1998;90:675-84.

27 Lee JC, Wang ST, Lai MD, et al. K-ras gene mutation is a useful predictor of the survival of early stage colorectal cancers. Anticancer Res 1996; 16:3839-44

28 Samowitz WS, Holden JA, Curtin K, et al. Inverse relationship between microsatellite instability and K-ras and p53 gene alterations in colon cancer. Am J Pathol 2001;158:1517-24.

29 Brink M, de Goeij AFPM, Weijenberg MP, et al. K-ras oncogene mutations in sporadic colorectal cancer in The Netherlands Cohort Study. Carcinogenesis 2003;24:703-10.

30 Keyse SM. Protein phosphatases and the regulation of mitogen-activated protein kinase signalling. Curr Opin Cell Biol 2000;12:186-92.

31 Management of Colorectal Cancer-A National Clinical Guideline. Scotland: Scottish Intercollegiate Guidelines Network (SIGN), 2003.

32 UK Colorectal Cancer Screening Pilot Group. Results of the first round of a demonstration pilot of screening for colorectal cancer in the United Kingdom. BMJ 2004;329:133-7.

33 Hardcastle JD, Chamberlain JO, Robinson MHE, et al. Randomised controlled trial of faecal-occult-blood screening for colorectal cancer. Lancet 1996;348:1472-7.

34 Sachse C, Smith G, Wilkie MJ, et al. A pharmacogenetic study to investigate the role of dietary carcinogens in the etiology of colorectal cancer. Carcinogenesis 2002;23:1839-49. 